\title{
Impacto da intervenção educacional sobre suicídio na percepção de enfermeiras e agentes comunitários de saúde*
}

Francielle de Rezende Rocha ${ }^{1}$

(D) https://orcid.org/0000-0003-2837-1164

Marcia Regina Martins Alvarenga ${ }^{2}$

(D) https://orcid.org/0000-0003-1367-6475

Bianca Cristina Ciccone Giacon-Arruda ${ }^{1}$

(D) https://orcid.org/0000-0002-8433-6008
* Este artigo refere-se à chamada temática "Violência autoprovocada: autolesão não suicida e comportamento suicida".

* Artigo extraído de dissertação de mestrado "Intervenção educacional com enfermeiros e agentes comunitários de saúde sobre suicídio", apresentada à Universidade Federal de Mato Grosso do Sul, Campo Grande, MS, Brasil.

${ }^{1}$ Universidade Federal de Mato Grosso do Sul, Instituto Integrado de Saúde, Campo Grande, MS, Brasil.

2 Universidade Estadual de Mato Grosso do Sul, Dourados, MS, Brasil.
Objetivo: analisar a percepção de enfermeiras e agentes comunitários de saúde em relação ao suicídio antes e após uma intervenção educativa. Método: estudo quase experimental, do tipo antes e depois, de abordagem qualitativa, baseado no referencial teórico da Transferência de Conhecimento. Participaram do estudo duas enfermeiras e dez agentes comunitários de saúde de uma unidade básica de saúde de Campo Grande, do estado de Mato Grosso do Sul. Foi utilizada a técnica de grupo focal, para a coleta de dados, antes e após a intervenção educativa. A análise dos dados foi realizada por meio da técnica de Triangulação de Métodos. Resultados: emergiram duas categorias da análise dos dados, "a percepção do suicídio nos seus diferentes aspectos", que traz percepções, compreensões e conceitos relacionados ao suicídio e "o contexto da assistência à saúde no comportamento suicida", que mostra como o suicídio é vivenciado pelos profissionais de saúde nos espaços de cuidado. Conclusão: a intervenção educativa favoreceu mudanças na percepção dos participantes referentes à identificação e abordagem do comportamento suicida, indicando a importância de ações dessa natureza para abordagens inerentes à prevenção do suicídio.

Descritores: Suicídio; Estudo de Intervenção; Atenção Primária de Saúde; Tentativa de Suicídio.

\section{Como citar este artigo}

Rocha FR, Alvarenga MRM, Giacon-Arruda BCC. Impact of the educational intervention about suicide on the perception of nurses and community health agents. SMAD, Rev Eletrônica Saúde Mental Álcool Drog. 2020;16(4):13-22. doi: https://dx.doi.org/10.11606/issn.1806-6976.smad.2020.168225 


\section{Impact of the educational intervention about suicide on the perception of nurses and community health agents}

Objective: to analyze the perception of nurses and community health agents in relation to suicide before and after an educational intervention. Method: a quasi-experimental research, of the before and after study type and with a qualitative approach, based on the theoretical framework of Knowledge Transfer. Two nurses and ten community health agents from a basic health unit in Campo Grande, state of Mato Grosso do Sul, participated in the study. The focus group technique was used for data collection before and after the educational intervention. Data analysis was performed using the technique of Triangulation of Methods. Results: two categories of data analysis emerged: "the perception of suicide in its different aspects", which brings perceptions, understandings, and concepts related to suicide; and "the context of health care in suicidal behavior", which is characterized by how suicide is experienced by the health professionals in care contexts. Conclusion: the educational intervention fostered changes in the participants' perception regarding the identification and approach of suicidal behavior, indicating the importance of actions of this nature for approaches concerning suicide prevention.

Descriptors: Suicide; Intervention Study; Primary Health Care; Suicide Attempted.

\section{Impacto de la intervención educativa sobre el suicidio en la percepción de las enfermeras y agentes comunitarios de salud}

Objetivo: analizar la percepción de enfermeras y agentes comunitarios de salud en relación con el suicidio, antes y después de una intervención educativa. Método: estudio cuasi experimental, del tipo antes y después, con enfoque cualitativo, basado en el marco teórico de la Transferencia de Conocimiento. Los participantes del estudio fueron dos enfermeras y diez agentes comunitarios de salud de una unidad básica de salud de Campo Grande en el estado de Mato Grosso do Sul. Se utilizó la técnica del grupo focal se utilizó para recopilar datos antes y después de la intervención educativa. El análisis de los datos se realizó mediante la técnica del Método de Triangulación. Resultados: surgieron dos categorías de análisis de datos, "la percepción del suicidio en sus diferentes aspectos", que trae percepciones, entendimientos y conceptos relacionados con el suicidio y "el contexto de la atención en salud en el comportamiento suicida", que muestra cómo los profesionales de la salud experimentan el suicidio en los espacios de atención. Conclusión: la intervención educativa favoreció cambios en la percepción de los participantes con respecto a la identificación y el enfoque del comportamiento suicida, lo que indica la importancia de acciones de esta naturaleza para abordajes inherentes a la prevención del suicidio.

Descriptores: Suicidio; Estudio de Intervención; Atención Primaria de Salud; Intento de Suicidio. 


\section{Introdução}

O suicídio é um grave problema de saúde pública global e está entre as vinte principais causas de morte em todo o mundo. No período de 2010 a 2016, a taxa global de suicídios padronizada por idade diminuiu $9,8 \%$, com reduções que variam de $19,6 \%$ na região do Pacífico Ocidental a $4,2 \%$ na região do Sudeste Asiático. Entretanto, houve um aumento de $6 \%$ dessas taxas na Região das Américas, no mesmo período(1).

No Brasil, no período de 1996 a 2015, houve crescimento de óbitos nas regiões norte, nordeste e sudeste, diminuição na região Sul e tendência estável na região centro-oeste. Na região sul foi criado, em 2009, um programa de prevenção do suicídio, fortalecendo a atenção básica e incluindo a identificação de riscos em capacitações profissionais. Reforçando, assim, a necessidade do desenvolvimento de ações educativas que contemplem a divulgação dos riscos, programas de prevenção e complicações relacionadas ao suicídio, com prioridade na atenção básica de saúde(2).

Frente a isso, a realidade brasileira ainda indica uma fragilidade na implantação de um programa nacional de vigilância e de estratégias efetivas de prevenção do suicídio, para o enfrentamento a esse problema de saúde pública. Investimentos para formação dos profissionais que assistem às pessoas com comportamento suicida (CS) e aos seus familiares são imprescindíveis, principalmente para uma prestação adequada de assistência e cuidado em saúde ${ }^{(3)}$.

Sabe-se, também, que muitos profissionais de saúde ainda têm atitudes negativas em relação ao indivíduo com CS. Isso demonstra a relevância do desenvolvimento de programas de capacitação, que contemplem, além do conhecimento, mudanças nas atitudes dos profissionais de saúde que favoreçam a empatia e compreensão do CS, por parte de todos os profissionais de saúde(4).

Destarte, intervenções educativas sobre o suicídio podem melhorar atitudes dos membros da equipe de saúde, contribuindo para o desempenho adequado do papel a ser executado na assistência em saúde(5). A mudança de percepção sobre a assistência e atuação do enfermeiro e agentes comunitários de saúde (ACS) diante da pessoa com CS é uma ferramenta para prevenção do suicídio(6).

Frente a isso, questiona-se: A intervenção educacional em prevenção e posvenção do suicídio para enfermeiros e ACS pode favorecer mudanças de percepção e atitude em relação ao tema e sua assistência? Assim, o objetivo desse estudo foi analisar a percepção de enfermeiras e ACS em relação ao suicídio, antes e após uma intervenção educativa.

\section{Método}

Estudo quase-experimental, do tipo antes e depois, de abordagem qualitativa, baseado no referencial teórico da Transferência de Conhecimento(7). A abordagem qualitativa foi escolhida devido ao objetivo de analisar a percepção e atitudes em relação ao suicídio, antes e depois uma intervenção educativa.

Para o desenvolvimento da intervenção educativa desse estudo, foi utilizado o modelo teórico de transferência de conhecimento denominado como processo "Knowledge-to-Action" (KTA) que tem como finalidade direcionar a aplicação do conhecimento. $O$ KTA define que todos os usuários finais do conhecimento são atores importantes no processo de transferência de conhecimento, o qual ocorre em duas fases simultâneas: a fase da criação do conhecimento e a fase da ação(7). Esse modelo de referencial foi escolhido por permitir analisar a percepção dos participantes sobre o CS e construir, em conjunto, possibilidades de melhorias que poderiam ser aplicadas na assistência desse contexto.

O local do estudo foi uma unidade básica de saúde (UBS) de Campo Grande, do estado de Mato Grosso do Sul, composta por três equipes de estratégia de ACS, constituídas, cada uma, por uma enfermeira e dez ACS. A unidade foi escolhida devido a sua proximidade territorial com o Centro de Atenção Psicossocial (CAPS), referência de atendimento a indivíduos com ideação e tentativas de suicídio, fator que seria um facilitador para o matriciamento entre as unidades de atendimento.

Como participantes do estudo, foram convidados todos os enfermeiros e ACS das três equipes, somando um total de 30 ACS e 3 enfermeiros. O convite aos enfermeiros foi por intermédio da gerente da unidade e, dos ACS, pessoalmente pela pesquisadora principal. Como critério de inclusão, definiu-se profissionais enfermeiros e ACS com período de atuação, no local da pesquisa, igual ou superior a um mês (período que permite que o ACS tenha uma compreensão de suas atribuições e da comunidade). Foram excluídos os profissionais que se encontravam de férias ou afastados do trabalho no período da realização do estudo.

A intervenção educativa foi conduzida apenas pela pesquisadora principal. A carga horária total foi de 24 horas, dividida em oito encontros semanais com duração de três horas cada. O conteúdo programático foi desenvolvido por meio de metodologias ativas e abordou o conceito de suicídio, epidemiologia, fatores protetores e de risco para o suicídio, abordagem profissional, avaliação e manejo do CS, e ações de prevenção e posvenção do suicídio.

Para a coleta de dados, foram utilizados um questionário sociodemográfico e uma entrevista por meio da técnica do grupo focal. O questionário sociodemográfico foi elaborado pelas próprias autoras e 
continha dados referentes à profissão, sexo, faixa etária, escolaridade, tempo de lotação na unidade e possíveis participações em cursos, seminários, congresso e/ou palestras sobre o tema suicídio.

O grupo focal foi escolhido como método de coleta de dados por sua técnica subsidiar a aplicação do referencial teórico KTA. Trata-se de uma técnica relevante que permite que os participantes sejam sujeitos ativos na construção dos dados, fomentando a aproximação entre os cenários de prática e as pesquisas ${ }^{(8)}$.

Os grupos focais foram realizados por duas pesquisadoras treinadas, ambas enfermeiras, sendo uma a pesquisadoras principal, a qual conduziu o grupo, e outra que fez o registro da observação. Os grupos foram realizados um encontro antes e um encontro depois dos encontros da intervenção educativa, e conduzidos por meio de questões norteadoras: O que você pensa a respeito da pessoa que tenta suicídio? O que você acredita que facilita a identificação da pessoa com CS? Como profissional de saúde, qual a sua atitude perante uma pessoa com CS? Como você conversaria com os familiares de uma pessoa que tentou suicídio?

O período entre o primeiro grupo focal, a intervenção educativa e o último grupo focal foi de setembro a outubro de 2018. O material foi gravado em mídia digital de áudio, transcrito na íntegra e organizado para a análise. A análise se deu por meio da Análise pela Triangulação de Métodos, de forma indutiva, que tem como propósito articular as informações obtidas com a pesquisa(9).

A primeira articulação foi a transcrição e avaliação dos dados para a elaboração de categorias para análise, que são os dados empíricos (os relatos abordados no grupo focal). Na segunda etapa da articulação, realizou-se uma revisão das evidências científicas existentes sobre o objeto para contextualização. $\mathrm{Na}$ terceira etapa, a análise de conjuntura, avaliou-se o objeto do estudo relacionado à ambiência social, articulando os dados coletados com as normatizações do assunto.

Para finalizar, foi feita uma reinterpretação, envolvendo dados empíricos, evidências científicas sobre o tema e a análise de conjuntura emergida do conhecimento local para o campo mais amplo de pesquisa. As falas dos grupos focais serão apresentadas com a sigla A - ACS e E - enfermeiros, e divididas em momento 1 (pré-intervenção) e momento 2 (pós-intervenção).

O estudo foi desenvolvido de acordo com as diretrizes para realização de pesquisas com seres humanos vigentes ${ }^{(10)}$. Ele foi aprovado pelo Comitê de Ética em Pesquisa com Seres Humanos da Universidade Federal de Mato Grosso do Sul (UFMS), CAAE:
83059318.0.0000.0021, e todos os participantes assinaram o Termo de Consentimento Livre e Esclarecido.

\section{Resultados}

Dos 33 profissionais de saúde, um total de duas enfermeiras e dez ACS aceitaram participar do estudo. Deles, dois ACS desistiram de participar do estudo, durante o desenvolvimento, por razões particulares.

Assim, dos 10 participantes, dois eram enfermeiras e oito ACS, sendo $83 \%$ do sexo feminino, $50 \%$ tinham idade entre 31 e 45 anos, 33\% entre 46 e 55 anos e $17 \%$ entre 25 e 30 anos. Quanto à escolaridade, $42 \%$ tinham ensino médio completo, $25 \%$ ensino superior, $17 \%$ tinham especialização. Em relação ao tempo de atuação na unidade participante do estudo, metade dos participantes já estavam lotados no local há mais de 4 anos, e metade a menos de 2 anos. Apenas 33\% dos participantes já haviam tido contato prévio com o tema suicídio por meio de palestra e/ou seminários.

A partir da análise dos dados, emergiram duas categorias: "a percepção do suicídio nos seus diferentes aspectos" e "o contexto da assistência à saúde no CS".

\section{A percepção do suicídio nos seus diferentes aspectos}

Essa categoria descreve as percepções, compreensões e conceitos relacionados ao suicídio presentes nos grupos focais antes da intervenção educativa e as mudanças percebidas depois a intervenção.

Antes da realização da intervenção educativa (momento 1), o suicídio foi descrito pelos participantes como um ato que representa sofrimento, muitas vezes devido ao indivíduo não conseguir lidar com problemas, por estar cansado, por não conseguir enfrentar situações conflituosas, ou como um ato de desespero.

...que ela não vê saída, que ela comete. Ela não vê uma saída plausível para aquela situação. Ela não quer se matar, ela quer se livrar do problema. Então, ela não vê uma solução para se livrar do problema, e acaba cometendo...(A10) momento 1

Para os participantes, os indivíduos que têm risco para suicídio demonstram ou aparentam tristeza e sofrimento. Dessa maneira, eles descrevem uma dificuldade na compreensão e identificação do CS em pessoas que fogem desse estereótipo, que têm suporte social e espiritual, e sem problemas de saúde.

Eu tenho um colega só, que se matou. Um colega, extremamente alegre. Por isso que eu te falo que essa experiência de ver a pessoa chorando, aquela depressão mais visível não. Extremamente alegre, no mesmo dia a gente estava junto, tudo feliz da vida. Nunca, nunca, nada, sabe. E ele se matou, se enforcou...não tinha vício, não tinha nada. (A9) momento 1 
Essa percepção implica na ideia de que algumas pessoas que tentam suicídio o fazem para chamar a atenção da família e das pessoas à sua volta. Assim, o comportamento de pessoas que ameaçam se suicidar, com frequência é percebido como atitudes de alguém que não irá cometer suicídio. Frases de alertas não consideradas pelas famílias ou outras pessoas também foram mencionadas nos grupos focais, indicando que não acreditavam que os indivíduos teriam coragem.

...que a gente tem que vencer, é uma dificuldade mesmo. É esse preconceito que a gente tem, quando chega algum caso, a gente fala...ah, só está querendo chamar atenção... A gente nunca pensa no real motivo que provocou aquilo...(E1) momento 1

Há uma ambivalência, expressada pelos participantes, sobre a compreensão do suicídio. Os mesmos vinculam o ato de se matar a um ato de coragem, por conseguir se matar, ou a um ato de covardia, pelo não enfrentamento de seus problemas.

...mas aquela pessoa que comete suicídio, ela se torna tão corajosa de acabar com a própria vida. Porque tem que ter coragem e ele se torna tão covarde por não querer enfrentar isso. E, ele pensa que é o inverso, que ele é um covarde, não enfrentar a situação da vida que está passando, mas mesmo ele se torna um corajoso...(A2) - momento 1

Entretanto, a análise das narrativas do grupo focal depois a intervenção educativa, indicou outra maneira de perceber o suicídio pelos participantes. A impulsividade presente no CS é indicada como um dos fatores para o ato, não havendo mais a descrição como um ato de coragem ou covardia. Houve a compreensão de que diferentes transtornos mentais, e não apenas a depressão, são fatores importantes no CS.

É o impulso, não é a coragem. Se passou esse momento de impulso, eles não se matam mais. Eu acho que depois de tudo que nós vimos aqui no curso, não é coragem ou fraqueza... (A5) - momento 2.

Em relação ao direito de se matar, mesmo depois da intervenção educativa, os participantes permaneceram expressando o conflito sobre se apenas Deus tem o direito sobre a vida. Entretanto, depois da intervenção educativa, aparece o questionamento sobre a situação da pessoa com CS estar doente e com a capacidade de tomar decisões alterada. E, a crença que a espiritualidade possa servir como apoio para esses indivíduos.

...por exemplo, toda pessoa tem direito de tirar a própria vida. Nós tivemos aquele questionamento antes, de que a pessoa não está com saúde mental dela adequada, obviamente, ela não tem esse direito. Porém, também existe, é direito dela enquanto dono de si...(A9) - momento 2

Em relação ao estigma e ao preconceito do adoecimento mental, foi referida pelos participantes a percepção de que as pessoas não refletem sobre os motivos para o suicídio. Eles associaram essa dificuldade ao estigma e ao tabu de falar sobre o assunto, e à crença de que falar sobre o assunto pode influenciar no CS. Após a intervenção educativa, o preconceito e a dificuldade de se falar sobre o suicídio entre outros colegas profissionais de saúde foi associado à falta de conhecimento e capacitação.

...em todos os níveis de saúde, desde o agente. Igual a (A3) falou, com a enfermeira. Eu vou levar você, às vezes é preconceito, às vezes é falta de capacitação...(A5) - momento 2

\section{O contexto da assistência à saúde no CS}

Essa categoria se caracteriza pela forma que o suicídio é vivenciado pelos profissionais de saúde nos espaços de cuidado. Foi possível também perceber, nos relatos, a modificação nessa percepção após a intervenção educativa.

Para os participantes, antes da intervenção educativa, em função de os sinais e sintomas do CS serem específicos, apenas um especialista poderia ser capaz de identificar e reconhecer. Após a intervenção, eles referiram à importância de se atentar às frases de alerta que uma pessoa com CS possa utilizar, e entender que uma pessoa aparentemente feliz pode ter ideação suicida, mesmo sendo mais difícil identificar.

... realmente, essas pessoas que tentam, a maioria das vezes, elas dão sinais. Geralmente que elas estão pensando em fazer alguma coisa, pode ser pelo comportamento, pelas atitudes, pela conversa da pessoa, então eu acho que a gente tem que estar preparado para saber identificar isso...(E1) momento 2

Antes da intervenção educativa, referiram não se sentirem preparados para atender alguém com CS. E afirmaram que, devido a isso, postergam investigações de notificações de tentativas de suicídio. Nesse contexto de assistência, relataram acreditar que a insistência no assunto pode romper o vínculo com a família e o indivíduo. Estratégias de abordar família compartilhando problemas pessoais ou por meio da religião foram descritas.

Eu estou vendo as fichas lá de violência e eu tenho que ir. Como eu vou abordar? Eu ainda não sei, por isso que a gente as guarda. Eu sei que eu tenho que pegar firme naquelas fichas, porque eu sei que tem uma ficha de uma moça que já tentou quatro vezes e entre as tentativas de suicídio, tem as violências domésticas que ela vem sofrendo. O que eu faria é encaminhar ela para o psicólogo, ela ir para o CAPS e começar o tratamento, até aí eu vou. (E2) - momento 1; Mas esse negócio de conversar, de abordar. Uma coisa é você contar os seus problemas, a sua experiência, os seus problemas pessoais e mostrar que não tem só o meu problema, o problema dela, todo mundo tem problema. É uma boa forma de abordar, quando a pessoa abre brecha. (A6) - momento 1

Após a intervenção educativa, os relatos indicaram que os participantes se sentiram mais preparados 
para fazer abordagens e dialogar com pessoas que apresentam CS, famílias e outros usuários, sem o receio de incitar pensamentos e CS, e sem usarem perguntas automáticas, focadas somente nas condições físicas. Compreenderam que exercem um papel importante no manejo de situações de aflição e sofrimento. Entretanto, referiram receio de se envolverem emocionalmente.

Porque eu achava que não podia fazer pergunta. Porque a pessoa está querendo se matar, a pesquisadora esclareceu que não, que é importante, às vezes, perguntar o que a pessoa pensa. Me sinto mais preparada depois do curso...(A3) momento 2 .

Em relação à família, antes da intervenção educativa, referiram que deveria ficar a critério do profissional de saúde abordar ou não a família, ou indivíduo sobre o assunto, principalmente quando havia história de tentativas ou de suicídio. Porém, indicaram que a família que vivencia esse contexto tem um sofrimento intenso, reconhecendo a importância de ajudar a família a compreender o CS e o suicídio.

Se a pessoa ainda não se matou, aí você tem que fazer a família tentar entender por que ela está ameaçando se matar. E quando a pessoa não se matou é assim. Agora, quando a pessoa já se matou, aí tem que falar que não é culpa de ninguém, que a pessoa estava fugindo de um problema, tem que fazer a pessoa entender por que o ente querido dela se matou. (A3) momento 1

Antes da intervenção educativa, os participantes referiram sobre ações a serem mediadas com moradores, a respeito do CS, que poderia haver encaminhamentos pela unidade básica de saúde, como psicólogos, psiquiatras e formação de grupos familiares como rede de apoio. Entretanto, com ações centradas apenas na figura do profissional médico e/ou psicólogo.

...eu encaminharia para um psicólogo ou para um psiquiatra. O enfermeiro pode encaminhar para o psicólogo... (E2) - momento 1

Contudo, após a intervenção educativa, relataram a importância de assistir esse indivíduo e sua família, durante todo o processo, ajudando a família e o indivíduo na compreensão do que estão vivenciando. E, também, a importância do trabalho com os sobreviventes do suicídio. Como sugestões de assistência, exemplificaram com a possibilidade de criação de grupo de familiares de moradores que passaram por situações semelhantes na área de abrangência da unidade.

...esclarecer para família que o suicida não se matou, nem por falta de amor à família e nem porque ele não amava a família , mas segundo o estudo que a gente fez, não é isso, quando a família consegue entender isso, é mais um conforto, uma libertação. (A5) - momento 2; É um grupo de apoio...a gente tem mais de uma família que poderia formar um grupo desse, poderia começar com apoio de um psicólogo e depois, talvez continuar sem psicólogo. (E2) - momento 2
Expuseram, antes da intervenção educativa, que capacitações sobre o tema podem ajudá-los a sentirem mais segurança para abordar o assunto e saber quais atitudes relacionadas podem adotar e quais ofertas de acompanhamento o Sistema Público de Saúde oferece.

...cursos assim, como esse para nós, já que nós estamos ali, cara a cara com a ponta como falam, não valorizam. Cursos como esses para saber lidar, identificar uma pessoa que está querendo se suicidar, o que aquela família está precisando naquele momento, que o filho ou alguém se suicidou. A gente poder falar com um pouco mais de propriedade do assunto em si. (A9) - momento 1

Em relação a esse tema, após a intervenção educativa também referiram o preconceito com o assunto do suicídio entre os próprios profissionais de saúde e que isso poderia ser uma barreira que dificultaria a abordagem sobre a temática. Associaram o preconceito à falta de capacitação.

Não é porque é profissional de saúde que é livre de preconceito. Então dentro dos profissionais de saúde, mesmo da própria enfermagem, a gente vê muito preconceito. Lá na UPA $X$, a gente escuta muitas coisas que doem o coração, de colegas, de técnicos, então assim, eu acho mais difícil conversar com os profissionais, tentar convencê-los...(E2) - momento 2

Para eles, questões sobre o suicídio deveriam ser elucidadas desde a infância e adolescência, no processo de formação escolar e que não há abordagem do tema suicídio no processo de formação universitária e que se sentem despreparados para receberem demandas sobre o assunto.

Eu não sou uma pessoa muito preparada para lidar com isso. Até pela minha formação, porque na faculdade, eu não lembro de nenhum momento ter tratado isso, alguma grade do meu curso. É falho, até certo ponto...(E1) - momento 1

Em relação ao sistema de saúde, referiram a morosidade para conseguir atendimento para as necessidades dos moradores, inclusive do morador com risco para o suicídio. Percebem má vontade dos profissionais em acompanhar os casos, e que quando encaminhados ou indicado a necessidade de encaminhamentos, não recebem devolutiva sobre os casos.

Aí que cai nesse negócio da assistência pública, ter o problema do atraso, não ter o especialista, sempre tem um problema também, a pessoa vai lá e fala, tem o CAPS, tem o posto para te ajudar, a pessoa vai lá e não consegue, isso atrapalha também. (A6) - momento 1

\section{Discussão}

Pode-se identificar que antes da intervenção educativa, tinham dúvidas e questionamentos sobre o suicídio. As explicações das causas do CS eram pautadas na influência da mídia e de fatores sociais, em estigmas 
e preconceitos do adoecimento mental, e associações com doenças e religião.

O estigma e os pré-conceitos, podem interferir na percepção do suicídio e na maneira em que o profissional de saúde promove o cuidado a indivíduos com CS. Ou seja, incertezas e indecisões nas condutas dos profissionais de saúde podem estar relacionadas a esse desconhecimento do tema, aos estigmas, pré-conceitos e mitos como o de falar sobre o suicídio pode favorecer pensamentos de morte ou que quem fala sobre o suicídio não irá se matar(11).

A pouca compreensão de aspectos subjetivos que envolvem uma tentativa de suicídio gera uma reação negativa da equipe de saúde frente ao indivíduo, podendo causar prejuízos nos cuidados oferecidos ${ }^{(12)}$. Dessa maneira, deve-se ampliar as discussões sobre suicídio durante o curso de graduação de enfermagem, podendo a inclusão deste tema gerar mudanças nos sentimentos e atitudes dos alunos ${ }^{(13)}$. Um estudo demonstrou que graduandos de enfermagem têm baixa exposição educacional específica no tema do suicídio, identificando a importância de desenvolver saberes atitudinais e não somente saberes cognitivos e procedimentais para intensificar a inserção de atitudes mais positivas ${ }^{(14)}$.

Ressalta-se que os profissionais de enfermagem podem realizar atividades educativas com o intuito de promover o conhecimento sobre o tema e estimular a tolerância, o respeito e a aceitação das diferenças. E, com isso, atitudes acolhedoras e compreensivas devem ser adotadas nos cuidados de enfermagem para ajudar a diminuir o impacto do estigma relacionados a transtornos mentais( ${ }^{(15)}$.

A religião também pode influenciar comportamentos e crenças e, com isso, pode haver aumento do estigma em torno do suicídio e da procura por ajuda profissional(16). Algumas religiões estão modificando a forma de abordar os transtornos mentais e suicídio, mas capacitações sobre o tema devem ser ofertadas a religiosos ${ }^{(17)}$.

Nesse estudo, no momento após a intervenção educativa, a espiritualidade é mencionada como fator protetor ao suicídio, como fonte de apoio à pessoa com CS. A literatura também indica que a fé pode ser um fator de proteção para o suicídio e que a espiritualidade pode ajudar muitas pessoas a seguir a vida durante uma crise $^{(16)}$.

Em relação à associação da expressão de tristeza e sofrimento ao CS, ou a um comportamento para chamar atenção, sabe-se que pessoas que cometeram suicídio demonstraram sinais de suas intenções. Dessa maneira, a presença de planos de suicídio caracteriza grau moderado a grave de risco, e menções sobre o suicídio sempre devem ser consideradas, principalmente pelos profissionais de saúde ${ }^{(11,16)}$.

Após a intervenção, notou-se que essa ligação a estereótipos de comportamentos e aparência física foram substituídas pela compreensão de sofrimento psíquico e pela percepção da necessidade de fornecer apoio ao indivíduo com CS e aos seus familiares. Houve o reconhecimento do comportamento impulsivo como fator de predisposição para o ato, em substituição à ambivalência entre coragem ou fraqueza. E, com isso, reflexão sobre o direito de se matar.

Estudo com objetivo de avaliar uma intervenção educativa, por meio de um curso de extensão universitária sobre CS, com estudantes de enfermagem, identificou que, após a realização do curso, houve mudança nos fatores relacionados aos sentimentos negativos e à percepção da capacidade profissional. Entretanto, para aquele relacionado ao direito de cometer o suicídio, não houve alterações. Ele demonstrou que a capacitação ofertada pode gerar mudanças positivas nas atitudes dos estudantes de enfermagem frente ao $\mathrm{CS}^{(18)}$.

A falta de conhecimento e preparação para lidar com o tema e sua influência na postergação de investigações de notificações de tentativas de suicídio também foi referida pelos participantes. Capacitações que abordem questões relacionadas aos cuidados em saúde mental são importantes, sendo relevante a qualificação contínua para o atendimento do paciente que tenta suicídio, para que estes não sejam submetidos à estigmatização e à falta de empatia, interferindo na qualidade do atendimento(12).

Outro fator importante versa sobre a influência das mídias. Evidências têm indicado que a internet, como mídias sociais, websites e salas de bate papo podem facilitar o acesso ao conteúdo sobre suicídio, acesso a informações sobre métodos, identificação, encorajamento, cyberbullying e, até mesmo, transformar o ato do suicídio em espetáculo, com encorajamento de pactos suicidas, principalmente entre adolescentes ${ }^{(16,19)}$.

Entretanto, as mídias podem ser usadas como uma ferramenta de proteção para os indivíduos, para promoção de saúde mental e prevenção do suicídio, com recursos como grupos de apoio, aplicativos, serious games e sites, sendo que em determinadas situações, o anonimato pode auxiliar na busca de ajuda $^{(19,20)}$.

Os resultados desse estudo também indicaram que a atenção primária à saúde (APS) ainda não é percebida como porta de entrada para atendimento do indivíduo com CS. E que os profissionais não se sentiam capazes de contribuir na prevenção e posvenção do suicídio. Entretanto, após a intervenção educativa, os participantes demonstram conhecimento sobre a 
necessidade de avaliar risco para o suicídio, para decidir as possíveis medidas a serem tomadas.

Em outro estudo também não foi possível identificar ações organizadas no processo de trabalho desse profissional que possibilitassem identificar fatores de risco associados ao suicídio na APS. A falta de treinamento seria um dos motivos possíveis para ausência de ações de prevenção neste contexto(21).

Devido ao contato próximo com as famílias pelas equipes da APS, estas são consideradas a primeira estratégia para as atividades de prevenção do suicídio, bem como acolhimento, identificação de risco e organização do cuidado propostos(16). Essas dificuldades dos profissionais de enfermagem e ACS, no cuidado e na classificação do grau de risco do CS, afetam os cuidados prestados, assim como a qualidade dos encaminhamentos para a especialidade em saúde mental(22).

Nesse sentido, o Ministério da Saúde lançou uma Agenda de Ações Estratégicas visando a vigilância e a prevenção do suicídio no Brasil, que contempla entre outros itens, a inserção do assunto suicídio na qualificação dos profissionais das portas de entrada do SUS, implementando dessa forma, a diretriz Acolhimento da Política Nacional de Humanização(23).

A necessidade de buscar soluções através de encaminhamentos ao serviço especializado, a acessibilidade e a morosidade na continuidade de prestação de serviços foram preocupações evidenciadas pelos participantes. Contudo, na pós-intervenção, evidenciou-se a percepção de que todos os profissionais de saúde devem estar aptos para prestarem assistência a esses indivíduos e suas famílias.

Em um estudo, usuários não percebem articulação entre serviços da atenção básica e serviços específicos de saúde mental e referem à necessidade de maior contato com outros equipamentos de seus territórios. Ao entrar no CAPS, sentem que saíram definitivamente da rede de saúde e percebem poucas parcerias entre os CAPS e a atenção básica, com as demandas de saúde mental centralizadas nos serviços especializados $^{(24)}$.

Com o objetivo de verificar as atitudes de profissionais da APS diante do CS, estudo concluiu que a capacitação efetiva da equipe para identificação precoce e tratamento apropriado para prevenção de suicídio, e tentativas de suicídio, é uma opção viável que contribui para diminuir os coeficientes de mortalidade por suicídio(4).

Foi referida, após a intervenção educativa, a importância do trabalho com os sobreviventes do suicídio, a necessidade da interação com a família e de se formar uma rede de apoio. A vivência entre enlutados por suicídio é importante estratégia de prevenção e posvenção do suicídio, em que experiência em grupos se torna um ambiente de elaboração do luto de uma perda trágica e ensinamentos sobre pertencimento e o acolher sem julgar ${ }^{(25)}$. O treinamento de profissionais de saúde durante a graduação, pós-graduação e em serviço é essencial para lidar com alguém que deseja legitimar sua dor psicológica(26).

$\mathrm{Na}$ pós-intervenção, o receio de se tornar responsável pela vida da pessoa com CS é expresso. O profissional precisa entender a diferença entre ser responsável pelo tratamento de alguém com ideação suicida e ser responsável pela vida desta pessoa. Conversas sobre prevenção do suicídio com o indivíduo, profissionais e outras pessoas envolvidas nos cuidados é uma forma de dividir preocupações e compartilhar o sentimento de se sentir responsável pela vida de alguém(16,27).

\section{Conclusão}

Esse estudo permitiu analisar a percepção de enfermeiras e agentes comunitários de saúde em relação ao suicídio antes e após uma intervenção educativa, e as mudanças ocorridas. A intervenção educativa promoveu a ampliação da compreensão do CS, facilitando a identificação de comportamentos de riscos e sinais de alerta, e desenvolvendo a capacidade de investigar o grau da intencionalidade do suicídio, assim como o oferecimento de apoio aos familiares da pessoa com ideação suicida.

Além disso, foi possível identificar a fragilidade na formação dos profissionais frente ao tema do suicídio e seu manejo na população, e do sistema público de saúde quanto à acessibilidade e continuidade da assistência.

Percebeu-se que a capacitação sobre suicídio pode ser um facilitador para o acesso do indivíduo com CS na APS, possibilitando melhorias no processo de trabalho e na assistência, assim como na articulação da rede de atenção.

Acredita-se que esse estudo possa contribuir com as evidências sobre a importância do ensino, de propostas de capacitação, formação e educação permanente dos profissionais de saúde. E na sensibilização de gestores, profissionais de saúde e educadores sobre a importância da aquisição de conhecimento para formação de uma rede de apoio para colaborar na prevenção e posvenção do suicídio, principalmente APS.

Como limitação, o estudo foi desenvolvido com apenas uma unidade de saúde em um território específico. Ressalta-se a necessidade de novos estudos de intervenção educativa para melhor compreensão do processo de transferência de conhecimento desse tema e estabelecimento de processos educativos mais eficazes e efetivos na melhora da assistência prestada. 


\section{Referências}

1. World Health Organization. Suicide in the world. Global health estimates. Geneva: WHO, 2019. Available from: https://www.who.int/publications-detail/ suicide-in-the-world.

2. D'Eça AE Júnior, Rodrigues LS, Meneses EP Filho, Costa LLN, Rêgo AS, Costa LC, Batista, RFLB. Mortality by suicide in the Brazilian population, 1996-2015: what's the predominant trend? Cad Saúde Coletiva. 2019; 27 (1):20-2. doi: 10.1590/1414-462X201900010211.

3. Silva DA, Marcolan JF. Epidemiology of suicide in Brazil between 1996 and 2016 and the public policy. Res Soc Develop. 2020; 9 (2): e79922080. doi: http://dx.doi. org/10.33448/rsd-v9i2.2080.

4. Storino BD, Campos CF, Chicata LCO, Campos MA, Matos MSCM, Nunes RMCM, Vida CEL. Attitudes of health professionals toward suicidal behavior. Cad Saúde Coletiva. 2018; 26 (4):369-77. doi: 10.1590/1414-462X201800040191.

5. Ramberg IL, Lucca MA, Hadlaczkly G. The Impact of Knowledge of Suicide Prevention and Work Experience among Clinical Staff on Attitudes towards Working with Suicidal Patients and Suicide Prevention. Int J Environ Res Public Health. 2016; 13:195. doi: https://doi. org/10.3390/ijerph13020195.

6. Magalhães CA, Neves DMM, Brito LMDM, Leite BBC, Pimenta MMF, Vidal CEL. Attitudes of medical Students toward Suicide. Rev Bras Educ Médica. 2014; 38(4):470-6. doi: https://doi.org/10.1590/ S0100-55022014000400008.

7. Graham ID, Tetroe JM. The Knowledge to Action Framework. In: Rycroft Malone, J.; Bucknall, T. Models and frameworks for implementingevidence-based practice: Linking evidence to action. WestSussex-UK: Wiley-Blackwell; 2010. p. 207-221.

8. Backes DS, Colomé JS, Erdmann RH, Lunardi V.L. Grupo focal como técnica de coleta e análise de dados em pesquisas qualitativas. O Mundo da Saúde. 2011; 35 (4):438-42.

9. Marcondes NAV, Brisola EMAB. Analyis by Triangulation of Methods: A Rererence to Qualitative Research. Rev Univap. 2014; 20 (35). doi: http://dx.doi.org/10.18066/ revunivap.v20i35.228.

10. Resolução no 466, de 12 de dezembro de 2012 (BR). Diário Oficial da União, Poder Executivo, Brasília, DF, 13 jun. 2013. Seção 1, 59. Disponível em: https://www.jusbrasil.com.br/diarios/55483111/ dou-secao-1-13-06-2013-pg-59.

11. Bertolote JM. O suicídio e sua prevenção. 1th ed. Unesp, 2012.

12. Fontão MC, Rodrigues J, Lino MM, Kempfer SS. Nursing care to people admitted in emergency for attempted suicide. Rev Bras Enferm. [Internet].
2018; 71 (suppl 5): 2329-35. doi: http://dx.doi. org/10.1590/0034-7167-2017-0219.

13. Silva LLT. Atitudes e percepções de estudantes e professores de enfermagem frente ao suicídio de adolescentes [tese]. Programa de Pós graduação em Ciências da Saúde da Faculdade de Medicina da Universidade de São Paulo; 2014. Disponível em: https://repositorio.ufmg.br/handle/1843/BUBD-9VZJ4H. 14. Moraes SM, Magrini DF, Zanetti ACG, Santos MA, Vedana KGG. Attitudes and associated factors related to suicide among nursing undergraduates. Acta Paul Enferm. 2016; 29 (6):643-9. doi: http://dx.doi. org/10.1590/1982-0194201600090.

15. Vedana KGG, Silva DRAS, Miasso AI, Zanetti ACG, Borges TL. The Meaning of Stigma for People with Mental Disorders in Brazil. Issues Mental Health Nurs. 38(12):1022-9. Doi: doi.org/10.1080/01612840.2017. 1346013.

16. Botega NJ. Crise suicida: avaliação e manejo. Porto Alegre: Artmed; 2015.

17. Scavacini K. O suicídio é um problema de todos: a consciência e a competência e o diálogo na prevenção e posvenção do suicídio [tese]. Instituto de Psicologia da Universidade de São Paulo. São Paulo; 2018. doi: 10.11606/T.47.2018.tde-26102018-155834.

18. Botti NCL, Araúlo LMC, Costa EE, Machado JSA. Nursing students attitudes across the suicidal behavior. Invest Educ Enferm. 2015; 33(2): 17-24. doi: 10.17533/ udea.iee.v33n2a16.

19. Pereira CCM, Botti NCL. Suicide in the communication of virtual social networks: Integrative literature review. Rev Portuguesa Enferm Saúde Mental. 2017; (17), 17-24. doi: http://dx.doi.org/10.19131/rpesm.0179.

20.Vedana KGG. Social mediais and suicide. SMAD, Rev. Eletrônica Saúde Mental Álcool Drog. 2018; 14(4): 194195. doi: 10.11606/issn.1806-6976.smad.2018.152315. 21. Silva NKN, Carvalho CMS, Magalhães JM, Carvalho JAM Júnior, Sousa BVSS, Moreira WC. Nursing actions in primary care to prevent suicide. SMAD, Rev Eletrônica Saúde Mental Álcool Drog. 2017; 13 (2): 71-7. doi: 10.11606/issn.1806-6976.v13i2p71-77.

22. Silva PF, Nóbrega MPSS, Oliveira, E. Knowledge of the nursing team and comumunity agentes on suicide behavior. J Nurs UFPE on line. 2018; 12(1): 112-7. doi: https://doi. org/10.5205/1981-8963-v12i01a23511p112-117-2018. 23. Ministério da Saúde (BR). Agenda de ações estratégicas para a vigilância e prevenção do suicídio e promoção da saúde no Brasil [Internet]. Brasília: BVSMS; 2017. Available from: http//bvsms.saude. gov. br/publicacoes/acoes_estrategicas_vigilancia.

24. Moreira MIB, Campos RTO. Mental health care actions in the psychosocial care network viewed by users. Saúde Soc. 2017; 26 (2): 462-74. doi 10.1590/ S0104-12902017171154. 
25.Scavacini K, Cornejo ER, Cescon LF. Support Group to the Bereaved by Suicide: an experience of postvention and social support. Revista M. 2019; 4 (7). doi: http:// dx.doi.org/10.9789/2525-3050.2019.v4i7.201-214.

26.Santos JC. Suicide: can we prevent the most mysterious act of the human being? Rev Portuguesa Enferm Saúde Mental. [Internet]. 2015; [Acesso 28 maio 2020]. 2(edição especial):7-8. Disponível em: www.scielo.mec.pt/scielo.php?pid=S164721602015000100001\&script=sci_arttext\&tIng=en.

27. Botega NJ. A tristeza transforma, a depressão paralisa. São Paulo: Benvirá; 2018.

\section{Contribuição dos autores}

Concepção e planejamento do estudo: Francielle de Rezende Rocha, Marcia Regina Martins Alvarenga e Bianca Cristina Ciccone Giacon-Arruda. Obtenção dos dados: Francielle de Rezende Rocha, Marcia Regina Martins Alvarenga e Bianca Cristina Ciccone Giacon-Arruda.

Análise e interpretação dos dados: Francielle de Rezende Rocha, Marcia Regina Martins Alvarenga e Bianca Cristina Ciccone Giacon-Arruda. Redação do manuscrito: Francielle de Rezende Rocha, Marcia Regina Martins Alvarenga e Bianca Cristina Ciccone Giacon-Arruda.

Revisão crítica do manuscrito: Francielle de Rezende Rocha, Marcia Regina Martins Alvarenga e Bianca Cristina Ciccone Giacon-Arruda.

Todos os autores aprovaram a versão final do texto.

Conflito de interesse: os autores declararam que não há conflito de interesse.
Recebido: 30.03.2020

Aceito: 26.05 .2020
Copyright $\odot 2020$ SMAD, Rev. Eletrônica Saúde Mental Álcool Drog. Este é um artigo de acesso aberto distribuído sob os termos da Licença Creative Commons CC BY-NC.

Esta licença permite que outros remixem, adaptem e criem a partir do seu trabalho para fins não comerciais, e embora os novos trabalhos tenham de lhe atribuir o devido crédito e não possam ser usados para fins comerciais, os usuários não têm de licenciar esses trabalhos derivados sob os mesmos termos. 